\title{
Traffic Grooming Algorithm Using Shortest EDPs Table in WDM Mesh Networks*
}

\author{
Seungsoo Lee, Tae-Jin Lee, Min Young Chung, and Hyunseung Choo \\ Lambda Networking Center, \\ School of Information and Communication Engineering, \\ Sungkyunkwan University, \\ 440-746, Suwon, Korea \\ \{isoolee, tjlee, mychung, choo\}@ece.skku.ac.kr
}

\begin{abstract}
In optical networks with huge transmission capability, Wavelength Division Multiplexing (WDM) has been actively studied in the research community. Traffic grooming technology based on divided bandwidth of a wavelength by WDM is very important for the network cost. This paper proposes a traffic grooming algorithm that employs the table for shortest Edge Disjoint Paths (EDPs) with clever selection on demands. Comprehensive simulations in various network environments show that the proposed algorithm outperforms well-known Maximizing Resource Utilization (MRU) up to $15 \%$ for the network throughput and up to $17 \%$ for the running time.
\end{abstract}

\section{Introduction}

Due to the explosive growth of hosts, users and services for the Internet, high speed network technologies based on optical networks have been appearing. Nowadays, Internet data traffic is increasing rapidly by more than $100 \%$ every year and in actuality, data traffic is passing existent voice traffic. Therefore existing networks cannot correspond with next generation data demand and capacity. Hence, in this context, research on optical technologies is drawing more interest for future high-speed network infrastructures [1].

Routing and wavelength assignment (RWA) for WDM networks, which are based on wavelength granularity, has been generally investigated [2]-4]. As WDM technology continues to mature, there exists a bandwidth difference between the transmission capacity (e.g., OC-48, OC-192, OC-768) and typical connection requests with low-late traffic stream (e.g., OC-1, OC-3, OC-12, etc.) 5. If the entire bandwidth of a wavelength channel is allocated to a low-late traffic stream, a large portion of the transmission capacity might be wasted. In order to effectively use network resources, many low-rate traffic streams should be efficiently groomed or multiplexed into a ligthpath [6].

\footnotetext{
* This work was supported in parts by Brain Korea 21 and the Ministry of Information and Communication, Korea. Corresponding author: Prof. H. Choo.
} 
This paper deals with the traffic grooming problem in WDM mesh networks with limited number of wavelengths for the environment of static traffic requests. Our objective is to maximize the network throughput. Since the optical network resource is limited, to maximize a throughput by increasing utilization rate of wavelengths is very important in terms of cost. The proposed algorithm works as follows. For a given network, it finds the shortest Edge Disjoint Paths (EDPs) 22 for all source and destination pairs and saves them to the shortest EDPs table (EDPT). It then calculates a resource utilization rate per hop for each request. Finally, it chooses a request with the biggest value of resource utilization rate, and sets the path corresponding to the selected request based on the information in EDPT. From simulation results, network throughput of the proposed algorithm is increasing up to about $10 \%$ in NSFNET and up to about $15 \%$ in random networks compared with that of the previous MRU algorithm. In operation time, the proposed algorithm is about $17 \%$ faster than the previous MRU in NSFNET.

The rest of the paper is organized as follows. In section 2, previous works on WDM SONET ring and mesh WDM networks, single-hop grooming and multi-hop grooming, and routing method for traffic grooming are presented. In section 3, we propose a traffic grooming algorithm in WDM mesh networks and describe the algorithm with an illustrative example. In section 4 , the performance evaluations are presented and discussed. Finally, section 5 concludes the paper.

\section{Related Works}

\subsection{Previous Works in Various Environments}

Recently the type of optical transport network topologies is evolving from interconnected SONET/WDM ring to optical WDM mesh. The traffic grooming problem in optical WDM mesh networks has become an important area of research [6]. In [5], a generic graph model was proposed for provisioning multigranularity connections in multi-wavelength optical WDM networks. The authors presented that for different network optimization objectives, their corresponding route selection schemes can be easily applied to the graph model. In [6], a heuristic traffic grooming algorithm is proposed for static traffic environments; maximizing resource utilization (MRU) algorithm which is known to be effective.

In general, to solve the traffic grooming problem, it is assumed that traffic requests are carried through single-hop grooming or multi-hop grooming. In single-hop grooming an end-to-end connection consists of a single lightpath. This means that only end-to-end traffic grooming is allowed. On the other hand, in multi-hop grooming a connection consists of multiple lightpaths. Thus, a connection can be dropped at intermediate nodes and groomed with other low-capacity connections on different lightpaths before it reaches its destination node. It is expected that the multi-hop grooming leads to a higher throughput than achieved by single-hop grooming. 


\subsection{Routing for Traffic Grooming}

In general routing algorithms can be classified into three categories; fixed, adaptive, and fixed-alternate routing [5]. In fixed routing, each connection is always routed through a predefined route for a its source-destination pair. Fixed shortest path routing is an example of this approach. The shortest path for each source-destination pair is calculated offline using shortest path algorithms. If there are not enough resources to satisfy a connection request, it is blocked.

In adaptive routing, the route from a source to a destination node is chosen dynamically, as current network status. The network status depends on the connections served. For example, when a connection request arrives, the shortest path between its source and destination node is determined as the available resources in the network.

In fixed-alternate routing, multiple routes are determined when ever a connection request occurs. In this scheme, each node within the networks has a table to maintain information, including the order of multiple routes on a destination node. For example, these routes can be ordered as the first shortest path, the second shortest path, and so on. When a connection request occurs, its source node sequencelly attempts to establish a route based on the order information in the routing table, until it is successfully completed.

\section{Proposed Algorithm}

We propose a new heuristic algorithm for the traffic grooming problem. Define a network topology $G(N, E, W)$ for a given WDM optical network, where $N$ is the set of nodes, $E$ is the set of edges, $W$ is the set of wavelengths in one fiber (each fiber is assumed to have the same number of wavelengths) and the capacity of each wavelength is $C_{w}$. Let $D=\left\{d_{i}(s, t) \mid \forall s, t \in N\right.$ and $1 \leq i \leq$ $|D|\}$ denote the traffic request between same source node s and destination node t. And $C(d(s, t))$ denotes the aggregate capacity of traffic request between the node pair $d(s, t)$. Let $H(s, t)$ denote the hop distance on the physical topology between the node pair $(s, t)$. We define $R(s, t)=C(d(s, t)) / H(s, t)$ as the connection resource utilization value, which represents the average traffic per wavelength link. This quantity shows how efficiently resources have been used to carry traffic requests.

The MRU algorithm sorts all of the node pairs $(s, t)$ in a descending order according to network resource utilization $R(s, t)$ and put them into a list $L$, then attempts to assign lightpaths sequentially. But after a lightpath for one node pair is assigned, network resource utilization of the other node pairs changes since the network state on the physical topology changes. The MRU algorithm does not consider changed network resource utilization, hence this algorithm does not reflect practical network resource utilization. This method cannot be applied to the values $R(s, t)$, which were calculated at the beginning. Eventually it influences in network throughput.

Our proposed algorithm calculates every available shortest EDPs for each $d(s, t)$ pair, and stores them to table $A$. Similar to the MRU algorithm, all 


\begin{tabular}{|c|c|c|c|}
\hline Traffic & $d(s, t), C(d(s, t))$ & $H(s, t)$ & $R(s, t)$ \\
\hline 1 & $(0,2)$, OC-72 & 2 & 36 \\
\hline 2 & $(1,2)$, OC-32 & 1 & 32 \\
\hline 3 & $(1,4)$, OC-60 & 2 & 30 \\
\hline 4 & $(5,2)$, OC-24 & 1 & 24 \\
\hline 5 & $(5,3)$, OC-48 & 2 & 24 \\
\hline 6 & $(4,3)$, OC-24 & 1 & 24 \\
\hline 7 & $(2,4)$, OC-12 & 1 & 12 \\
\hline
\end{tabular}

(a) Set of connection requests, $L$

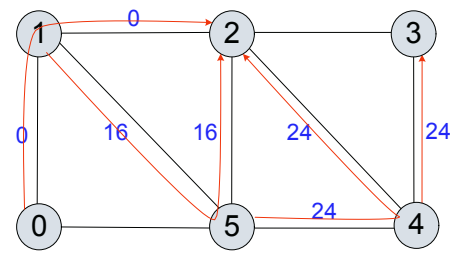

(c) MRU algorithm

\begin{tabular}{|c|c|}
\hline$(s, t)$ & Shortest EDPs \\
\hline$(0,2)$ & $0-1-2,0-5-2$ \\
\hline$(1,2)$ & $1-2$ \\
\hline$(1,4)$ & $1-2-4,1-5-4$ \\
\hline$(5,2)$ & $5-2$ \\
\hline$(5,3)$ & $5-2-3,5-4-3$ \\
\hline$(4,3)$ & $4-3$ \\
\hline$(2,4)$ & $2-4$ \\
\hline
\end{tabular}

(b) Shortest EDPT

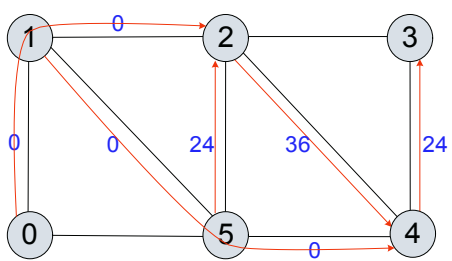

(d) Proposed algorithm

Fig. 1. Example of MRU and the proposed algorithm

of the node pairs $d(s, t)$ are sorted according to network resource utilization $R(s, t)$ between $(s, t)$ pairs and puts them into a list $L$ in a descending order. Then it finds the node pair with the highest $R(s, t)$ and attempts to assign a lightpath for this node pair $d(s, t)$. If there is enough resources in the network in one of the paths of table $A$, connection request can set up a lightpath. If there is not enough resources to establish a lightpath, the proposed algorithm tries the next connection request in list $L$. Therefore the values $R(s, t)$, which were calculated at the beginning, will be reflected to the decision of lightpaths and it can increase the throughput. Moreover, the proposed algorithm using the fixed-alternate routing method can expect fast routing than the MRU algorithm using adaptive routing.

We present an example for the proposed algorithm and the MRU algorithm for a simple network (Fig. 10. Let us assume that it is a single fiber WDM optical network with each fiber having one wavelength channel, and the capacity of wavelength is OC-48. The aggregated traffic for node pair $(0,2),(1,2),(1,4),(5,2)$, $(5,2),(4,3)$, and $(2,4)$ is $72,32,60,24,48,24$, and 12 , respectively. Those traffic requests are sorted by $R(s, t)$ and put into a list $L$ (Fig. 1(a)). Then it tries to setup a lightpath for the first node pair $d(s, t)$ in list $L$. Fig. $[1(\mathrm{c})]$ shows the setup procedure for a lightpath for in the MRU algorithm. We can see that connection requests of $1,2,4$, and 6 are accomodated according to the order of list $L$. In this case, overall network throughput is OC-128. On the other hand, our proposed algorithm tries to set up lightpaths based among the stored shortest EDPT (Fig. 1(b) . The network throughput with the proposed algorithm is OC-156. Which shows that proposed algorithm performs better than the MRU algorithm. 
The proposed algorithm can be divided into two parts and it employs both fixed-alternate routing and adaptive routing. Step 1 constructs a virtual topology: using fixed-alternate routing, it tries to establish lightpaths by the resource utilization values. Step 2 conducts traffic grooming on virtual topology: using adaptive routing, it tries to find out multi-hop routes for unassigned traffic requests. The procedure and pseudo-code for the proposed algorithm are as follows.

Step 1: Construct a virtual topology:

1.1: Aggregate traffic requests for each node pair $d(s, t)$ with the same $(s, t)$. $C(d(s, t))$ is the sum of unassigned capacity of traffic requests.

1.2: Compute all shortest EDPs and $H(s, t)$ for each node pair $d(s, t)$, and put them into a table $A$.

1.3: Compute $R(s, t)=C(d(s, t)) / H(s, t) \cdot R(s, t)$ is the connection resource utilization value

1.4: Sort all of the node pairs $d(s, t)$ according to $R(s, t)$ and put them into a list $L$.

1.5: Extract the element $d^{\prime}(s, t)$ with the greatest value from list $L$, and route according to shortest EDPs of table $A$. If it fails, delete $d^{\prime}(s, t)$ from $L$, otherwise, let $C\left(d^{\prime}(s, t)\right)=\max \left\{C\left(d^{\prime}(s, t)\right)-C_{w}, 0\right\}$

1.6: Go to step 1.5 until list $L$ is empty.

Step 2: Traffic grooming on virtual topology:

2.1: Satisfy all of the connection requests that can be carried through a single lightpath hop, and update the virtual topology network state.

2.2: Route the remaining connection requests using currently available spare capacity of the virtual topology based on their connection resource utilization value $R(s, t)$. If there is no route for $d(s, t)$, then reject this connection request.

\section{(Pseudo-code for Proposed Algorithm)}

Input: Network topology $G(N, E, W)$ and demand set

$$
D=\left\{d_{i}(s, t) \mid \forall s, t \in N \text { and } 1 \leq i \leq|D|\right\}
$$

Output: Total network throughput $T$

1. Find shortest EDPs for the demand set and store them in table $A$

2. Calculate $R(s, t)=C(d(s, t)) / H(s, t)$ for all demands

3. Sort all demands in descending order by $R(s, t)$ and store them in list $L$

4. For $(\lambda=1 ; \lambda \leq W ; \lambda++)$

5. // Construct a virtual topology

6. $\quad$ While $(L \neq \emptyset)$

7. $\quad$ Select $d^{\prime}(s, t)$ which is on top of $L$

8. If $\exists$ a path in $A$ for $d^{\prime}(s, t)$ then

9. Assign the path to $d^{\prime}(s, t)$ for the lightpath set up

10. $\quad C\left(d^{\prime}(s, t)\right)=\max \left\{C\left(d^{\prime}(s, t)\right)-C_{w}, 0\right\}$

11. If $C\left(d^{\prime}(s, t)\right)=0$ then delete $d^{\prime}(s, t)$ from list $L$ 
12. $\quad$ Else delete $d^{\prime}(s, t)$ from the list $L$ and store to list $L^{\prime}$

13. $\quad$ End while

14. // Routing $d(s, t)$ on the virtual topology

15. Update the network state and restore list $L=L \cup L^{\prime}$

16. Route the remaining $d(s, t) \mathrm{s}$ on the current network state by multi-hop grooming

17. If $\lambda=W$ then

18. The remaining $d(s, t)$ in list $L$ is blocked

19. End for

20. $T=(($ Total demand capacity - Blocked demand capacity $) /$

Total demand capacity) $\times 100$

21. Return $T$

In the proposed algorithm, inputs are network topology $G(N, E, W)$ and demand set $D$, and an output is network throughput $T$. After finishing the single-hop grooming, update the topology then try to setup lightpaths for the remaining requests by using multi-hop traffic grooming. The algorithm continues until there are no more wavelengths. After using all of the wavelengths, the remaining requests are blocked.

\section{Performance Evaluation}

In this section we evaluate the performance of the proposed algorithm by computer simulation in terms of the network throughput that is successfully carried traffic capacity and the running time. For the comparison study, we compare our proposed method with the MRU. For the evaluation in the typical network, we use NSFNET which has 14 nodes and 21 links. Moreover we employ randomly generated networks structures because we want to test those two schemes properly in various network topologies. The random connection request model proposed in 6 to generate our incoming traffic matrices is utilized here. In our case, we assume the capacity of each wavelength is $\mathrm{OC}-48$, and allow the traffic bandwidth requests to be any of OC-1, OC-3, and OC-12. The traffic metrics are randomly generated that the numbers of connection requests on OC-1, OC3 , and OC-12 for all possible node pairs are generated as uniformly distributed random numbers between 0 and 16 , between 0 and 8 , and between 0 and 2 , respectively. We simulate 1,000 times for each network topology and this is exactly same as experiments done in [6]. We obtain a random network for the physical topology by specifying the number of nodes in the graph model $(N)$ and the probability of edge existence $\left(P_{e}\right)$ for any node pair 3 .

In this work, we define the network throughput as follows:

$$
T=\frac{\sum_{i=1}^{|D|} C\left(d_{i}(s, t)\right) \times S_{i}}{\sum_{i=1}^{|D|} C\left(d_{i}(s, t)\right)} \times 100(\%)
$$


where $S_{i}=1$ if connection request $i$ has been satisfied; otherwise $S_{i}=0$, and the total number of connection requests is $|D|$.

Fig. 2(a) shows the results of two algorithms compared and it demonstrates the relationship between the network throughput $(T)$ and the number of wavelengths. When the number of wavelengths is either 1 or 2 , the proposed scheme and the MRU show almost the same trends because network resources are not enough for total connection requests. However, as the number of wavelengths increases, the proposed scheme shows the better performance than the MRU. Specifically, it outperforms the MRU by $10 \%$ in terms of the network throughput when the number of wavelengths reaches to 10 through 15 . As you see, when the number of wavelengths is in between 22 and 25, our scheme and the MRU both show the similar results since network resources are quite enough for total connection requests.

As mentioned earlier we perform the simulation study for random networks in order to have the generality in network structures. Simulation environments are similar to those of the fixed NSFNET except the variable $P_{e}$ for the probability of edge existence. We consider $P_{e}$ values as 0.3 and 0.6 , and the number of nodes as 15 and 20 for the reasonable sizes of experiments. If $P_{e}$ is high, we create dense networks. Otherwise, we have sparse ones.

Fig. 2(b) shows that the network throughput versus the number of wavelengths for the 15-node random networks. As a whole, the proposed algorithm performs better than the MRU. When $P_{e}$ is 0.3 , the number of wavelengths needed per link is 20 for routing all connection requests. In the meantime, for $P_{e}=0.6$, the number of wavelengths required per link is 6 for accommodating all demands. Because, if $P_{e}$ is 0.6 , then the network has more edges connected to each node than the case of $P_{e}=0.3$. It means that the number of possible paths which handle the requests is increased, so they can manage requests with relatively less number of wavelengths. As a result, the proposed algorithm improves the throughput up to about $10 \%$ and $14 \%$ compared with the MRU for $P_{e}=0.3$ and $P_{e}=0.6$, respectively, in 15-node random networks.

Fig. 2(c) presents the same case for 20-node random networks. It shows quite dense networks for $P_{e}=0.6$ in 20-node random networks, and as expected there are more shortest EDPs. It provides a flexibility for identifying paths, and that is why the proposed algorithm shows the better performance than the MRU. Therefore the proposed one outperforms the MRU by $15 \%$ in terms of the network throughput for such dense networks.

Fig. 2(d) shows that the running time for the algorithm including the selection of EDPs and wavelength assignments for requests. We generate the sets of traffic matrices for demand sets. As the number of demand sets increases, we measure the time. If there are a small number of demand sets (i.e. sets of traffic matrices), the proposed algorithm takes more time to find the shortest EDPs and to construct the table for EDPs than the MRU. However, as the number of demand sets increases, it runs faster. The table for shortest EDPs is never changed as long as the physical network structure is not changed. The proposed algorithm references the table to set up the lightpaths, meanwhile the MRU finds 


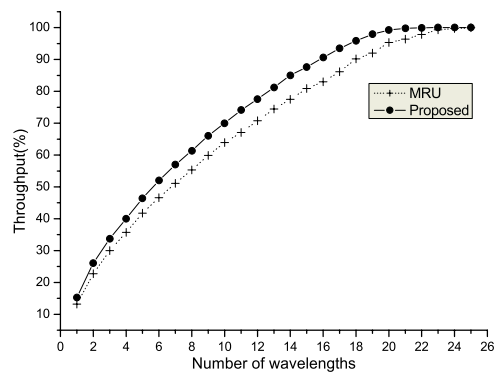

(a) Throughput in NSFNET

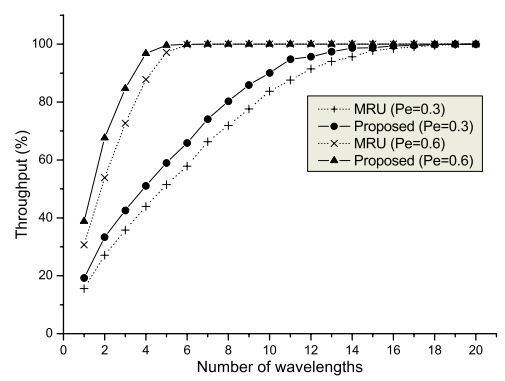

(c) 20-node random networks

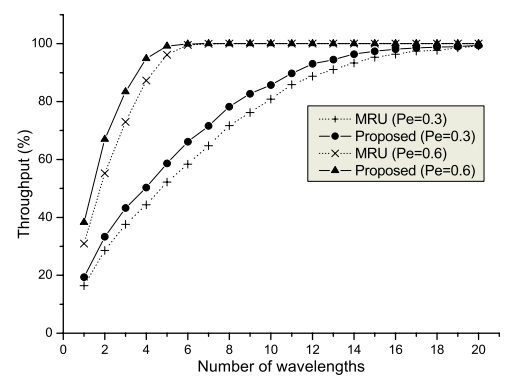

(b) 15-node random networks

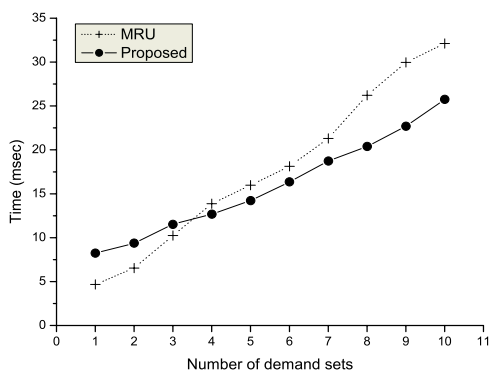

(d) Running time in NSFNET

Fig. 2. Results from the simulation study

a new shortest path in the current network state after setting up one request. If there are 10 sets of traffic matrices, it is about $17 \%$ faster than the MRU.

\section{Conclusion}

In this paper we study the traffic grooming problem in WDM mesh networks with the limited number of wavelengths for the environment of static traffic requests. The proposed algorithm is based on the table for shortest Edge Disjoint Paths (EDPs) with clever selections on demands. The objective of this work is to increase the network throughput and to decrease the running time as others do. Performance evaluations are done with fixed NSFNET and varied random networks. Comprehensive simulation shows that the proposed algorithm significantly improves the throughput up to $15 \%$ and the running time up to $17 \%$ compared to the previous MRU. This work will be applied to WDM multicasting networks in the near future. 


\section{References}

1. H. Choo, M. Son, M.Y. Chung and T.J. Lee, "Shared Protection by Concatenated Rings in Optical WDM Networks," Springer-Verlag LNCS, vol. 3042, pp. 1476-1482, May 2004.

2. P. Manohar, D. Manjunath and R.K. Shevgaonkar, "Routing and Wavelength Assignment in Optical Network From Edge Disjoint Path Algorithms," IEEE Communications Letters, vol. 6, pp. 211-213, May 2002.

3. M.H. Kim, H. Choo, B.H. Yae, J.H. Lee, "A Practical RWA Algorithm Based on Lookup Table for Edge Disjoint Paths," Springer-Verlag LNCS, vol. 2713, pp. 138147, June 2003.

4. H. Choo, and V.V. Shakhov, "Routing and Wavelength Assignments in Optical WDM Networks with Maximum Quantity of Edge Disjoint Paths," Springer-Verlag LNCS, vol. 3038, pp. 1138-1145, June 2004.

5. H. Zhu, H. Zang, K. Zhu, and B. Mukherjee, "A novel, generic graph model for traffic grooming in heterogeneous WDM mesh networks," IEEE/ACM Trans. Networking, vol. 11, pp. 285-299, Apr. 2003.

6. K. Zhu and B. Mukherjee, "Traffic grooming in WDM optical mesh networks," IEEE Journal on Selected Areas in Communications, vol. 20, pp. 122-133, January 2002. 